\title{
Detection of E6/E7 HPV oncogene transcripts as biomarker of cervical intaepithelial disolasia
}

\author{
Mauro Carcheri ${ }^{1}$, Caterina Oliveri ${ }^{1}$, Anna Fusco $^{1}$, Ilaria Chiossone ${ }^{1}$, Paola Milano ${ }^{1}$, \\ Annamaria Ferretti ${ }^{1}$,Anna Alabiso ${ }^{1}$, Licia Muselli' ${ }^{1}$, Rossana Cirillo ${ }^{2}$, Roberto Capuzzo ${ }^{1}$ \\ 1U.0. Laboratorio Analisi Chimico-Cliniche e Microbiologiche, \\ 2U.O. Ostetricia e Ginecologia - ASL 3 Genovese - Presidio Ospedaliero M etropolitano "Villa Scasi", \\ Corso Onofrio Scassi 1 - 16149 Genova
}

KeyWords: E6/E7 mRN A, HPV,ASC-US, LSIL, cervical neoplasia

\section{L'espressione delle proteine E6/E7 di HPV (PapillomaVirus Umano) quale marcatore di progres- sione verso forme severe di displasia intraepiteliale della cervice uterina}

\section{SUMMARY}

It is widely accepted that only persistent infection with high risk types of Human Papillomavirus (HPV HR) is a significant risk factor for the development of an invasive squamous cervical cancer.

The overexpression of viral oncogenes E6/E7 of H PV is considered a necessary process for incurring in a malignant phenotype.A HPV infection can be identified by detection of HPV DN A in biological samples, but the DN Abased tests cannot delineate between transient or persistent and potentially transforming infection. Instead there is many evidence to suggest that detection of HPV gene expression may constitute a more specific approach to highlight a clinically significant infection. Especially seems that the detection of E6/E7 transcripts can be usefully used for identify the women with a persistent HPV infection that will can induce a future cervical cancer.

The aim of our study is to investigate if the detection of oncogenic viral gene activity by detecting transcripts of the E6 and E7 genes can be most usefull of HPV-DN A test in the triage of ASC US or low grade cervical lesions. $\mathrm{O}$ ur results confirm that HPV E6/E7 mRN A test can be considered a promising method to stratify HPV positive women for risk of future high-grade cervical lesions or cervical intaepithelial neoplasia.

\section{Received June 6, 2009}

\section{INTRODUZIONE}

Nell'80.0\% dei casi il carcinoma della cervice uterina origina nel sottile strato di cellule piatte che delimitano il collo dell'utero e l'evidenza che il DNA del Papilloma Virus Umano (HPV) viene riscontrato nel 99.7\% dei carcinomi cervicali (35) ha indotto a riconoscerlo come la causa primaria, anche se non unica, di questo tipo di tumore solido. Il manifestarsi di modificazioni cellulari anormali (displasia) nei tessuti del collo uterino precede, di solito, la comparsa delle cellule cancerogene. Tra gli almeno 15 tipi di HPV riconosciuti ad alto rischio oncogeno (HPV-HR), i tipi virali HPV 16 e HPV 18 risultano di gran lunga quelli più frequentemente implicati nella genesi del carcinoma cervicale, essendo considerati responsabili rispettivamente di circa il $60 \%$ e il $10 \%$ di tutti i tumori cervicali (6) (17) (26). Nello sviluppo del cervicocarcinoma hanno rilevanza (anche se minore)
Accepted July 15, 2009

anche i tipi virali HPV 31, HPV 33, HPV 45 (19) (27). La maggior parte delle donne contrae un'infezione da HPV entro pochi anni dall'inizio dei rapporti sessuali. Il $75-80 \%$ di queste infezioni risultano transitorie, regrediscono spontaneamente per il coinvolgimento attivo del sistema immunitario del soggetto infettato e non presentano significato clinico anche se il risultato del Pap-test può mettere in evidenza alterazioni temporanee. (11) Solo in circa il 20\% dei casi l'infezione persiste più a lungo e può essere riscontrata anche $\mathrm{a}$ molti anni di distanza. Circa il 40\% delle infezioni "persistenti" ha come esito finale lo sviluppo di lesioni precancerose di alto grado o di un tumore della cervice uterina (2) (3) (10). E' ormai accertato che la trasformazione neoplastica delle cellule della cervice uterina è indotta dall'integrazione del DNA di HPV nel genoma della cellula ospite e dalla successiva produzione di due proteine

\section{Corresponding author: Mauro Carcheri}

U.O. Laboratorio Analisi - Presidio Ospedaliero Metropolitano "Villa Scassi" - ASL 3 Genovese

Corso O. Scassi, I - 16149 Genova - Tel: 010410239

E-mail: mauro.carcheri@villascassi.it 
codificate dai geni E6 ed E7 del virus (9). Solo l'infezione sostenuta da tipi di HPV in grado di produrre un'espressione deregolata delle oncoproteine E6 ed E7 è interessata nel processo di cancerogenesi e l'over-espressione di queste ultime, direttamente correlata con lo stato di proliferazione cellulare, è condizione necessaria per l'induzione ed il mantenimento del fenotipo maligno (33). La stretta correlazione tra infezione persistente e sviluppo di stati pre-cancerosi o cancerosi della cervice uterina ha fatto si che la ricerca del DNA di HPV HR in campioni di essudato cervicale assumesse, col tempo, sempre più rilevanza nella diagnostica preventiva del cervicocarcinoma (13) (21). Studi retrospettivi hanno messo in evidenza che l'infezione da HPV precede di molti anni la comparsa delle lesioni di alto grado e che le donne HPV negative hanno un minor rischio di sviluppare tali lesioni (4). Il Ministero della Salute ha inserito nelle "Raccomandazioni per la pianificazione e l'esecuzione degli screening di popolazione per la prevenzione del cancro della mammella, del cancro della cervice uterina e del cancro del colon retto" (2006) l'indicazione alla possibile esecuzione del test HPV nel triage delle donne con citologia ASCUS o LSIL in alternativa all'invio diretto in colposcopia e/o alla ripetizione della citologia a sei mesi. (21). Solo le donne positive al test HPV vengono inviate in colposcopia. Analogamente il Gruppo Italiano Screening del Cervicocarcinoma (GISCi) indica quale possibile test di approfondimento diagnostico il test HPV nel triage degli ASCUS e delle LSIL delle donne con più di 35 anni d'età e nel follow-up post trattamento per CIN 2 - CIN 3 e Adenocarcinoma endocervicale in situ (AIS) in quanto il test risulta avere importante valore predittivo negativo per CIN 3 e AIS. (13) Analoghe indicazioni sono riportate nelle raccomandazioni delle più importanti Società Scientifiche Ginecologiche (SIGO, AOGOI, AGUI, SICPCV) (1) (32). I test di biologia molecolare per la ricerca del DNA di tipi di HPV HR non sono però in grado di distinguere tra un' infezione che regredirà spontaneamente e un'infezione attiva, persistente e destinata a progredire (31). In particolar modo utilizzando i metodi basati sulla ricerca del DNA virale, si può giungere a riscontrare genotipi di HPV HR fino nel $20 \%$ del totale della popolazione femminile presa in esame, mentre, nella stessa popolazione $\mathrm{i}$ test istologici evidenziano una displasia significativa in meno dello $0.5 \%$ della stessa popolazione (20). Questa differenza è dovuta appunto al fatto che la maggior parte delle infezioni da HPV sono transitorie, con alti livelli di acquisizione ma anche di clearance mentre solo l'HPV che esprime attivamente le proteine oncogeniche E6 ed E7, può causare il cancro della cervice. (12) In definitiva si può affermare che il carcinoma della cervice uterina è un esito molto raro di una infezione molto comune (14). e che l'infezione da HPV precede di parecchio la comparsa di lesioni di alto grado, quando la citologia è ancora normale (16) (4).

In questa ottica un test in grado di rilevare i trascritti degli oncogeni E6 ed E7 di HPV, potrebbe possedere i requisiti per proporsi come vero e proprio marcatore di progressione verso la cancerogenesi (33) (34). Recenti studi di Kraus e coll. (19), Molden e coll. (22) (23) (24), Cuschieri e coll. (7) (8) (9) e altri Autori (5) (30) hanno determinato la sensibilità, la specificità, il Valore Predittivo Positivo (PPV) ed il Valore Predittivo Negativo (PNV) di questo test, concludendo che la ricerca dei trascritti di E6/E7 di HPV in associazione con la citologia, può risultare di grande aiuto nel triage delle donne con citologia ASCUS o LSIL in quanto, identificando le infezioni persistenti, consente di individuare più efficacemente le donne a maggior rischio di sviluppare un cervicocarcinoma.

Nel presente lavoro sono stati messi a confronto la tipizzazione virale basata sulla ricerca dell'HPVDNA tipo-specifico e il test di ricerca dell'mRNA delle oncoproteine E6 ed E7 di HPV, utilizzando un campione di donne per le quali era stato refertato un risultato citologico variabile da negativo a HSIL e si è cercato di valutare se il test basato sulla ricerca dei trascritti delle oncoproteine E6 ed E7 di HPV, inserito come test di approfondimento nello studio delle lesioni di basso grado e nel triage delle pazienti con lesioni pre-cancerose, possa effettivamente offrire un valore prognostico migliore rispetto al test basato sulla semplice ricerca dell'HPV-DNA.

\section{MATERIALI E METODI}

Sono stati presi in esame 214 campioni provenienti da donne per le quali risultava già nota una diagnosi citologica derivata da un Pap-test eseguito con tecnica tradizionale.

Per 40 di queste la diagnosi risultava negativa; per 70 era stato refertato un ASCUS; 62 avevano una diagnosi di LSIL, 42 di HSIL o superiore. L'età media delle donne prese in esame è risultata essere di 32 anni. Tutte le donne arruolate per il presente studio sono state sottoposte a prelievo per la ricerca di HPV-DNA (tipizzazione virale) e mRNA delle oncoproteine E6 ed E7. I campioni da sottoporre alle indagini molecolari per la ricerca di DNA e E6/E7 mRNA di HPV sono stati raccolti tramite un prelievo eseguito a livello endocervicale, ruotando il CytoBrush per tre volte in senso antiorario nel canale cervicale allo scopo di 
consentire una raccolta ottimale delle cellule. Il dispositivo di prelievo è stato poi stemperato per rotazione ed agitazione nella soluzione PreservCyt Solution del sistema di trasporto ThinPrep e quindi eliminato. Il contenitore del ThinPrep così preparato è stato trasferito immediatamente in laboratorio per l'estrazione degli acidi nucleici o conservato in frigorifero a $4^{\circ} \mathrm{C}$ sino al momento del processamento. Gli acidi nucleici (DNA e RNA) contenuti nei campioni così prelevati e conservati sono stati estratti utilizzando il dispositivo semi-automatico NucliSens miniMAG e i reattivi per l'estrazione (BioMerieux) basati sulla tecnologia di Boom a silice magnetica. Il DNA estratto è stato sottoposto ad amplificazione utilizzando 4 mix di primers diversi (per i tipi virali 6 e 11, per i tipi $16,31,33,35$, per i tipi $18,39,45,59$ e per quelli $52,56,58,66$ ) mediante una Multiplex-PCR che amplifica simultaneamente in una provetta un gruppo di target virali ed un Controllo Interno (IC: gene $ß$ globinico umano)

Il controllo di qualità (CQ) della seduta analitica è stato realizzato includendo un Controllo Positivo $(\mathrm{C}+)$ ed un Controllo Negativo (C-) in ognuna delle quattro sequenze di mix e, per i tipi virali HPV 16 e HPV 18 includendo anche quattro controlli esterni (QCE) costituiti da genoma completo di HPV 16 e HPV 18 clonato in plasmide e purificato mediante centrifugazione in gradiente

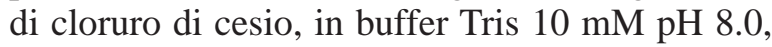
$1 \mathrm{~nm}$ EDTA. Per i due virus sono stati utilizzati campioni a basso titolo $\left(1 \times 10^{\wedge} 4\right.$ copie/ml $)$ e ad alto titolo $\left(1 \times 10^{\wedge} 7 \mathrm{copie} / \mathrm{ml}\right)$, nella quantità di 10 $\mathrm{ml}$ di campione in $200 \mathrm{ml}$ di siero negativo (Clonit S.r.l.) La rivelazione della presenza di amplificato specifico è stata eseguita mediante elettroforesi su gel di agarosio al $2 \%$ contenente $0.5 \mu \mathrm{g} / \mathrm{ml}$ di etidio bromuro. Il gel è stato fatto correre a 10 Volts/cm per circa 2 ore. Per evidenziare le bande corrispondenti ai diversi tipi virali è stata utilizzato un marcatore di pesi molecolari (50bp DNA Step Ladder) che evidenzia bande da 50 a 800 bp. A fine corsa il gel è stato posto su transilluminatore a UV (303 nm) e fotografato con filtro UV e pellicola Polaroid. Sono stati considerati positivi i campioni che contenevano le bande a 325 (HPV16), 520 (HPV31), 227 (HPV33) o 280 bp (HPV35) nell'amplificato con PCR mix-1 «1635» e le bande a 425 (HPV 18), 340 (HPV39), 475 (HPV45) o 395 bp (HPV395) nell'amplificato con PCR mix-1 «18-59», le bande a 360 (HPV52), 325 (HPV56), 240 (HPV58), 304 bp (HPV66) nell'amplificato con PCR mix-1 «52-66», le bande a 260 (HPV6), 425 bp (HPV11) nell'amplificato con PCR mix-1 «6,11».

Sono stati considerati negativi i campioni che contenevano solo la banda a 723 bp. (Controllo Interno) Nel caso di assenza della banda di 723 bp la seduta non è stata accettata in quanto l'assenza della banda dell'IC indica la non-idoneità del prelievo del campione. La sensibilità e la specificità cliniche dichiarate del test sono rispettivamente del 96.0\% e del 95.0\%. La sensibilità analitica del test è tale da consentire la rilevazione di circa 200 copie di HPV-DNA/ml Per la ricerca dell'mRNA delle oncoproteine E6 ed E7 è stato utilizzato il test NucliSENS EasyQ HPV della ditta bioMérieux che utilizza le tecnologie PreTect HPV-Proofer della NorChip e la NASBA RealTime di bioMérieux. Il test è in grado di rilevare la presenza di mRNA delle oncoproteine E6 ed E7 dei tipi virali HPV16, HPV18, HPV31, HPV33, HPV45, cioè dei tipi virali di HPV HR più frequentemente implicati nella genesi del cervicocarcinoma. L'integrità e l'adeguatezza dell'RNA, sono poste sotto controllo interno verificando l'amplificazione dell'mRNA del gene umano U1 che è un gene housekeeping poco abbondante. La riproducibilità del test è risultata all'interno di un range del 96-100\%. Il test E6/E7 mRNA ha una sensibilità clinica del $98.6 \%$ ed una specificità del 92.5\%.

Il Valore Predittivo Positivo (PPV) del test nei confronti di una possibile evoluzione a CIN2 o più nei casi di ASCUS o LSIL è del 37.5\% contro il $15.4 \%$ del test HPV-DNA mentre il Valore Predittivo Negativo (NPV) dei due test si equivale $(98.3 \%$ contro $97.1 \%)$. (24)

\section{RISULTATI}

Su 214 donne prese in esame, 130 (60.7\%) sono risultate negative alla ricerca di HPV-DNA (tipizzazione) e 84 (39.3\%) sono risultate positive Il test di ricerca dell'mRNA delle oncoproteine E6 ed E7 dei tipi virali HPV16, HPV18, HPV31, HPV33, HPV45 è risultato positivo in 42 casi (19.6\%) e negativo in 172 (80.4\%). (Tabella 1)

Il DNA di HPV 16 è stato rilevato, da solo o in coinfezione, in 54 casi su 84 risultati positivi (64.3\%), quello di HPV 18 in 12 casi (14.3\%), quello di altri genotipi di HPV in 18 casi (21.4\%). La ricerca dell'mRNA di E6/E7 è risultata positiva per il tipo virale HPV 16 in 32 casi su 42 positivi (76.2\%), per HPV 18 in 6 casi (14.3\%) e in 4 casi $(9.5 \%)$ per l'mRNA di altri tipi di HPV. (Tabella 2)

L'analisi risultati dei test di biologia molecolare per la ricerca di HPV in rapporto alla citologia fornisce questo quadro: (Tabella 3)

- Su 40 campioni derivati da donne con citologia negativa, 38 (95.0\%) sono risultati negativi al test di ricerca dell'HPV-DNA (tipizzazione) e 2 positivi (5.0\%). Nello stesso gruppo (citolo- 
gia negativa) il test di ricerca dell'mRNA di E6/E7 è risultato negativo per tutti e 40 i campioni presi in esame.

- I 70 campioni provenienti da donne con citologia ASCUS sono risultati negativi al test HPVDNA in 46 casi $(65.7 \%)$ e positivi allo stesso test in 24 casi (34.3\%).

In questo gruppo il test di ricerca dell'mRNA è risultato negativo per 66 campioni (94.3\%) e positivo per 4 (5.7\%).

- Nel caso dei 62 campioni provenienti da donne con citologia LSIL, i campioni negativi al test HPV-DNA sono risultati 30 (48.4\%), mentre quelli positivi sono risultati 32 (51.6\%). Il test di ricerca dell'mRNA è risultato negativo per 48 campioni (77.4\%) e positivo per 14 (22.6\%)

- I 42 campioni provenienti da pazienti con citologia HSIL o superiore sono risultati negativi al test HPV-DNA in 16 casi (38.1\%) e positivi allo stesso test in $26(61.9 \%)$

Con il test di ricerca dell'mRNA i negativi sono risultati 18 (42.9\%) ed i positivi 24 (57.1\%)

E' interessante, infine, notare che 2 dei 32 risultati positivi al test mRNA HPV E6/E7 (uno con espressione dell'mRNA di HPV31 e l'altro dell'mRNA di HPV45 ) sono relativi ai campioni di due pazienti con referto citologico di LSIL e che gli stessi campioni sono risultati negativi al test HPV-DNA. Risultati analoghi sono riportati anche da altri Autori (30).

\section{CONCLUSIONI}

I risultati, anche se ottenuti su di un campione numericamente limitato, confermano che la ricerca dell'mRNA delle oncoproteine E6 ed E7 di HPV HR mette in evidenza una percentuale di soggetti infettati da HPV più bassa (19.6\%) rispetto a quella rilevata dai test di ricerca dell'HPVDNA (39.3\%) (Tabella 1). La maggior parte delle positività messe in evidenza con il test di tipizzazione virale è dovuta alla rilevazione nel campione in esame di DNA del tipo virale HPV 16 (64.3\% dei casi), mentre HPV 18 si conferma il secondo tipo virale rilevato tramite il test di tipizzazione HPV-DNA. Anche tra i 32 risultati positivi ottenuti con il test di ricerca dell'mRNA delle oncoproteine E6/E7 di HPV, il tipo virale HPV 16 risulta quello più frequentemente riscontrato (76.2\% dei casi). (Tabella 2) L'esame dei risultati riportati in Tabella 3 conferma, infine, quanto ormai ampiamente riportato in letteratura e cioè che la ricerca dell'mRNA di E6/E7, al contrario dei test basati sulla ricerca del DNA virale, risulta positiva con maggiore frequenza in campioni di pazienti con citologia di grado superiore e meno nei casi di citologia negativa, ASCUS o LSIL.

I dati ottenuti con il test di tipizazzione HPVDNA mostrano infatti, nei casi di citologia ASCUS, una percentuale di positività pari al 34.3\% mentre, nello stesso gruppo, il test E6/E7 mRNA risulta positivo solo per una percentuale del 5.7\%. I risultati positivi ottenuti con il test di ricerca del DNA salgono al 51.6\% nei casi di LSIL e al $61.9 \%$ nei casi di citologia HSIL o superiore, mentre la percentuale di positività del test E6/E7 mRNA risulta decisamente più bassa nei casi di ASCUS (5.7\%) e appare in crescita dai casi LSIL (22.6\%) a quelli HSIL o più (57.1\%).

Tabella 1. Risultati (negativo e positivo) del test di tipizzazione (H PV-DNA) e del test di ricerca dell'mRN A delle oncoproteine E6/E7 sul totale dei 214 campioni presi in esame

\begin{tabular}{lll}
\hline & tipizzazione (HPV-DNA) & mRNA di E6/E7 \\
\hline NEGATIVO & $130(60.7 \%)$ & $172(86.0 \%)$ \\
\hline POSITIVO & $84(39.3 \%)$ & $42(19.6 \%)$ \\
\hline
\end{tabular}

Tabella 2. Prevalenza dei tipi virali (da soli o in co-infezione) sui positivi da Tabella 1

\begin{tabular}{llcc}
\hline & HPV 16 & HPV 18 & altri genotipi \\
\hline HPV-DNA (tipizzazione) & $54(64.3 \%)$ & $12(14.3 \%)$ & $18(21.4 \%)$ \\
\hline E6/E7 mRNA & $32(76.2 \%)$ & $6(14.3 \%)$ & $4(9.5 \%)$ \\
\hline
\end{tabular}

Tabella 3. Percentuali di positività rilevate dal test di tipizzazione (HPV-DNA) e dal test E6/E7 mRNA in relazione al risultato citologico (Pap-test tradizionale, refertazione secondo il Sistema di Bethesda 2001)

\begin{tabular}{llllll}
\hline & \multicolumn{5}{c}{ diagnosi citologica } \\
\cline { 2 - 6 } & & Negativa (40) & ASCUS (70) & LSIL (62) & HSIL o superiore (42) \\
\hline HPV-DNA & negativo & $38(95.0 \%)$ & $46(65.7 \%)$ & $30(48.4 \%)$ & $16(38.1 \%)$ \\
\cline { 2 - 6 } & positivo & $2(5.0 \%)$ & $24(34.3 \%)$ & $32(51.6 \%)$ & $26(61.9 \%)$ \\
\hline \multirow{2}{*}{ HPV E6/E7 } & negativo & $40(100.0 \%)$ & $66(94.3 \%)$ & $48(77.4 \%)$ & $18(42.9 \%)$ \\
\cline { 2 - 6 } & positivo & $0(0.0 \%)$ & $4(5.7 \%)$ & $14(22.6 \%)$ & $24(57.1 \%)$ \\
\hline
\end{tabular}


I risultati ottenuti, analoghi a quelli riportati in molti lavori già presenti in letteratura, sembrano confermare che l'espressione delle oncoproteine E6 ed E7 può realmente essere considerata un marker di progressione verso il cervicocarcinoma e che, pertanto, il test mRNA E6/E7 HPV potrebbe essere proficuamente utilizzato quale test di approfondimento nei casi di citologia dubbia o borderline.

In un prossimo futuro un nuovo schema operativo di prevenzione del cervicocarcinoma, che potrebbe essere proposto per donne di tutte le età potrebbe essere, pertanto, il seguente:

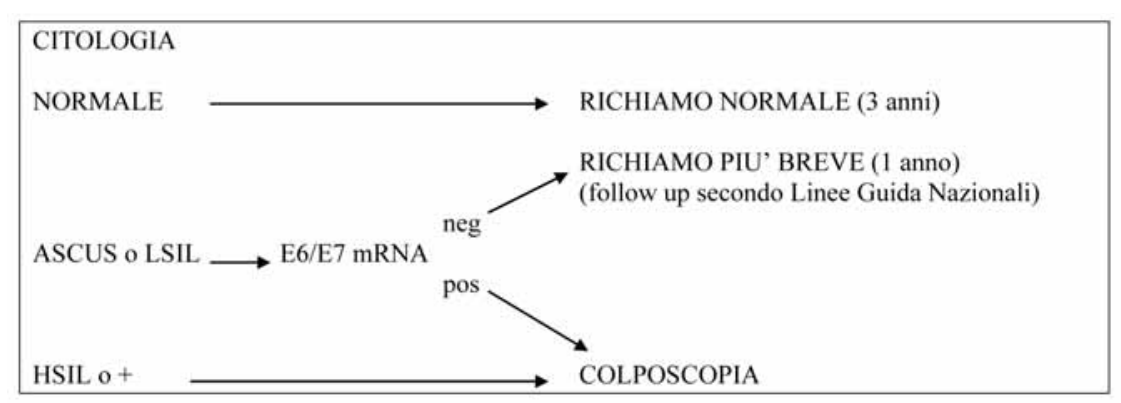

da il Triage dei risultati ASCUS/LSIL.

In particolare potrebbe portare ad una significativa diminuzione del numero di colposcopie, di biopsie e trattamenti non necessari con conseguente risparmio globale dei costi a fronte di un effettivo migliore trattamento del paziente ed a una sostanziale riduzione dello stress psicologico a carico delle donne con un'infezione "transiente".

Il test di ricerca dell' E6/E7 mRNA potrebbe risultare di grande utilità anche come test di approfondimento dei campioni HPV DNA positivi in caso tale test fosse adottato come screening primario, nel Follow-up dopo Trattamento, nello Screening e Follow-up di tumori Noncervicali e nel Follow-up della Vaccinazione.

In ultimo, anche se i genotipi virali presi in esame dal test E6/E7 mRNA (HPV16, HPV18, HPV31, HPV33, HPV45) sono di gran lunga quelli più frequentemente implicati nella genesi di

L'adozione di questo algoritmo potrebbe consentire di raggiungere significativi traguardi in termini di efficacia nell'opera di prevenzione del cervicocarcinoma consentendo contemporaneamente una ottimizzazione dei costi per quello che riguar-

\section{BIBLIOGRAFIA}

1. Atti $82^{\circ}$ Congresso Nazionale SIGO Roma ottobre 2006 (SIGO, AOGOI, AGUI, SICPCV)

2. Bosch FX, de SS. The epidemiology of human papillomavirus infection and cervical cancer. Dis.Markers 2007;23:213-27

3. Bosch FX, Lorincz A, Munoz N, et al. The causal relation between human papillomavirus and cervical cancer. J Clin Pathol 2002;55:244-65

4. Carozzi F, Ronco G, Confortini M, et al. Prediction of high grade cervical intraepithelial neoplasia in cytologically normal women by human papillomavirus testing. Br J Cancer. 2000; 83: 1462-1467

5. Cattani P, De Vincenzo R, Zannoni GF, et al.Comparison of HPV E6/E7 mRNA expression, DNA detection and cytological and histological findings in cervical samples. EUROGIN 2008 (abstracts) - Nice november 12-15 2008

6. Clifford GM, Smith JS, Plummer M, et al. Human papillomavirus in invasive cervical cancer worldwide: a meta-analysis. Br J Cancer 2003, 88:63-73

7. Cuschieri KS, Beattie G, Hassan S, et al. Assessment of human papillomavirus mRNA detection over time in cervical specimens collected in liquid based cytology medium. J Virol Methods. 2005 Mar;124(12):211-5

8. Cuschieri KS, Cubie HA. 2005 The roleof human papillomavirus testing in cervical screening. J.Clin.Virol. 32S:S34

9. Cuschieri K, Wentzensen N. Human Papillomavirus mRNA and p16 detection as biomarkers for improved diagnosis of cervical neoplasia. Cancer Epidemiol Biomarkers Prev 2008;17(10). October 2008 lesioni di alto grado rimane da stabilire se questo limitato numero di tipi virali è sufficiente per individuare effettivamente tutte le donne a rischio per il cervicocarcinoma.

10. Cuschieri KS, Whitley MJ, Cubie HA. 2004 Human papillomavirus type specific DNA and RNA persistence-implications for cervical disease progression and monitoring. J.Med.Virol. 73:65

11. Documento congiunto GISCi, SIGO, AOGOI. Lo screening organizzato del cervicocarcinoma: esigenze dei ginecologi coinvolti nello screening ed esigenze del programma di screening nei confronti dei ginecologi. (aggiornato 26/09/08) of acquisition and clearance of cervical human papilfor cervical cancer. J Infect Dis 1999;180:1415-23

13. GISCi: Utilizzo del test HPV HR nel triage delle diagnosi citologiche di atipia squamosa di significato indeterminato e delle diagnosi di LSIL in donne con piu’ di 35 anni nonche' nel follow-up delle lesioni cin2-3 come indicatore di rischio di recidiva (www.GISCi - aggiornato il 17/09/07)

14. IARC Press Release $N^{\circ} 151$ 03/05/2004)

15. IARC Working Group. Human Papillomaviruses. IARC Monographs on the evaluation of carcinogenic risks to humans,vol. 64. Lyon. International Agency for Research on Cancer; 1995

16. Ylitalo N, Sorensen P, Josefsson AM, et al. Consistent high viral load of human papilloma virus 16 and risk study. Lancet 2000;355:2194-8

17. Khan, MJ, et al. The elevated 10-year risk of cervical precancer and cancer in women with human papillomavirus (HPV) type 16 or 18 and the possible utility of type-specific HPV testing in clinical practice. Journal of the National Cancer Institute; 97: 14, 1072-1079. 2005
12. Franco EL, Villa LL, Sobrinho JP, et al. Epidemiology lomavirus infection in women from a high-risk area of cervical carcinoma in situ: A nested case-control 
18. Kleter B, et al. Development and clinical evaluation of a highly sensitive PCRreverse hybridization line probe assay for detection and identification of anogenital human papillomavirus. J Clin Microbiol.37(8):250817. 1999

19. Kraus I, Molden T, Holm R, et al. Presence of E6 and E7 mRNA from Human Papillomavirus Types 16, 18, 31, 33, and 45 in the Majority of Cervical Carcinomas. Journal Of Clinical Microbiology, Apr. 2006, p. 1310-1317 Vol. 44

20. Liverani CA, Mojana G. Infezione genitale da HPV: esami di laboratorio. La Colposcopia in Italia Anno $X X$ - N. 2 pagg. $12-16$ - ottobre 2005

21. Ministero della Salute Direzione Generale della Prevenzione "Raccomandazioni per la pianificazione e l'esecuzione degli screening di popolazione per la prevenzione del cancro della mammella, del cancro della cervice uterina e del cancro del colon retto" (2006)

22. Molden T, Kraus I, Karlsen F, et al. Comparison of Human Papillomavirus Messenger RNA and DNA Detection: A Cross-sectional Study of 4,136 Women $>30$ Years of Age with a 2-Year Follow-up of HighGrade Squamous Intraepithelial Lesion. Cancer Epidemiol Biomarkers Prev 2005;14(2). February 2005

23. Molden T, et al. PreTect HPV-Proofer: real-time detection and typing of E6/E7 mRNA from carcinogenic human papillomaviruses. J Virol Methods. 142(1-2):204-12. 2007

24. Molden T, Nygård JF, Kraus I, et al. and Hagmar B. Predicting CIN2 when detecting HPV mRNA and DNA by PreTect HPV-Proofer and consensus PCR: a 2-year follow-up of women with ASCUS or LSIL Pap smear. Int. J. Cancer: 114, 973-976 (2005)

25. J Möckel, A Clad, Endres AS, et al. HPV E6/E7 mRNA transcripts as predictors of high-grade epithelial cervix dysplasia. Diagnostic Pathology 2007, 2(Suppl 1):S1

26. Muñoz N, Bosch FX, Castellsague X, et al. Against which human papillomavirus types shall we vaccinate and screen? The international perspective. Int $J$ Cancer 2004; 111:278-85
27. Muñoz N, Bosch FX, de Sanjose S, et al. Epidemiological classification of Human papillomavirus types associated with cervical cancer. $N$ Engl J Med 2003;348:518-27

28. Nobbenhuis MAE, et al. Addition of high-risk HPV testing improves the current guidelines on follow-up after treatment for cervical intraepithelial neoplasia. Brit J Cancer 2001;84:796-801 Ronco G, Segnan N, Giorgio-Rossi P, et al, and the NTCC Working Group.

29. Human papillomavirus testing and liquid-based cytology in primary screening of women younger than 35 years: results at recruiment for a randomised controlled trial. Lancet Oncol 2006;7:547-55

30. Rosini S, Zappacosta R, Di Bonaventura G, et al. Management and triage of women with human papillomavirus infection in follow-up for low-grade cervical disease: association of HPV-DNA and RNA-based methods. Int.J.Immunopathol.Pharmacol. vol.20, no2,341-347 (2007)

31. Srodon M, Parry Dilworth H, Ronnett BM. Atypical squamous cells, cannot exclude high-grade squamous intraepithelial lesion: diagnostic performance, human papillomavirus testing, and follow-up results. Cancer. 2006 Feb 25;108(1):32-8

32. The Atypical Squamous Cell of Undetermined Significance/ Low-grade squamous Intraepithelial Lesions Triage Study (ALTS) Group: Human papillomavirus testing for triage of women with cytologic evidence of low-grade squamous intraepithelial lesions: baseline data from a randomized trial. J Natl Cancer Inst 2000, 92:397-402

33. Vocaturo A, Gustinucci D. Marcatori di progressione. in Papillomavirus - Dal Pap test ai vaccini. Certezze e prospettive. Analysis - N.1/2/3.2008 p.53-59

34. von Knebel Doeberitz M, Syrjanen KJ. Molecular markers: how to apply in practice. Gynecol.Oncol. 2006;103:18-20

35. Walboomers JM, Jacobs MV, Manos MM, et al. Human papillomavirus is a necessary cause of invasive cervical cancer worldwide. J Pathol. 1999 Sep;189(1):12-9 